\title{
Non contiguous-finished genome sequence and description of Peptoniphilus timonensis sp. nov.
}

\author{
Ajay Kumar Mishra ${ }^{1}$, Jean-Christophe Lagier ${ }^{1}$, Catherine Robert ${ }^{1}$, Didier Raoult ${ }^{1}$ and Pierre- \\ Edouard Fournier ${ }^{1 *}$ \\ ${ }^{1}$ Unité de Recherche sur les Maladies Infectieuses et Tropicales Emergentes, UMR, Aix- \\ Marseille Université, France \\ *Corresponding author: Pierre-Edouard Fournier (pierre-edouard.fournier@univmed.fr)
}

Keywords: Peptoniphilus timonensis, genome

\begin{abstract}
Peptoniphilus timonensis strain $\mathrm{JC} 401^{\top}$ sp. nov. is the type strain of $P$. timonensis sp. nov., a new species within the Peptoniphilus genus. This strain, whose genome is described here, was isolated from the fecal flora of a healthy patient. P. timonensis is an obligate Grampositive anaerobic coccus. Here we describe the features of this organism, together with the complete genome sequence and annotation. The 1,758,598 bp long genome ( 1 chromosome, no plasmid) contains 1,922 protein-coding and 22 RNA genes, including 5 rRNA genes.
\end{abstract}

\section{Introduction}

Peptoniphilus timonensis strain $\mathrm{JC401}{ }^{\mathrm{T}}$ (= CSUR P165= DSM 25367) is the type strain of $P$. timonensis sp. nov. This bacterium is a Grampositive, anaerobic, indole-positive coccus that was isolated from the stool of a healthy Senegalese patient as part of a "culturomics" study aiming at cultivating individually all species within human feces.

Since the early days of bacterial taxonomy, defining a bacterial species has been a matter of debate. Currently, the availability of a wide array of molecular methods, notably $16 \mathrm{~S}$ rRNA and full genome sequencing, offers a possibility to base the description of new species on other methods than the "gold standard" of DNA-DNA hybridization [1]. In particular, sequence similarity of the $16 \mathrm{~S}$ rRNA, although neither uniform across taxa nor necessarily predictive, enabled the taxonomic classification or reclassification of many taxa [2], and genome sequencing has provided access to the complete genetic information of bacteria [3]. As a consequence, we based our description of $P$. timonensis sp. nov. on a polyphasic approach [4] including their genome sequence and main phenotypic characteristics (habitat, Gram-stain reaction, culture and metabolic characteristics, MALDI-TOF spectrum, and when applicable, pathogenicity).
Here we present a summary classification and a set of features for $P$. timonensis sp. nov. strain JC401 ${ }^{\mathrm{T}}$ together with the description of the complete genomic sequencing and annotation. These characteristics support the creation of the $P$. timonensis species.

The genus Peptoniphilus (Ezaki et al. 2001) was created in 2001 [5] and consist of species that are non-saccharolytic, butyrate-producing, non-motile gram-positive anaerobic cocci and use peptones and oligopeptide as major energy source [6]. To date, the genus Peptoniphilus contains eight species namely $P$. asaccharolyticus, $P$. harei, $P$. indolicus, P. ivorii, P. lacrimalis [5], P. gorbachii, P. olsenii [6], P. methioninivorax [7]. Members of the genus Peptoniphilus have mostly been isolated from various human clinical specimens such as vaginal discharges, ovarian, peritoneal, sacral and lachrymal gland abscesses [5]. P. indolicus causes summer mastitis in cattle [5].

\section{Organism information}

A stool sample was collected from a healthy 16year-old male Senegalese volunteer patient living in Dielmo (rural village in the Guinean-Sudanian zone in Senegal), who was included in a research protocol. The patient gave an informed and signed 
consent, and the agreement of the National Ethics Committee of Senegal and the local ethics committee of the IFR48 (Marseille, France) were obtained under agreement (09-022 and 11-017). The fecal specimen was preserved at $-80^{\circ} \mathrm{C}$ after collection and sent to Marseille. Strain JC401 ${ }^{\mathrm{T}}$ was isolated in June 2011 by cultivation on 5\% sheep bloodenriched Brain Heart Infusion agar (Becton Dickinson, Heidelberg, Germany). This strain exhibited a $98 \%$ nucleotide sequence similarity with Peptoniphilus harei, the phylogenetically closest validated Peptoniphilus species (Figure 1, for classification, see Table 1). This value was lower than the $98.7 \%$ 16S rRNA gene sequence threshold recommended by Stackebrandt and Ebers to delineate a new species without carrying out DNADNA hybridization [18].

Table 1. Classification and general features of Peptoniphilus timonensis strain $\mathrm{JC} 401^{\top}$ according to the MIGS recommendation [8]

\begin{tabular}{|c|c|c|c|}
\hline MIGS ID & Property & Term & Evidence code $^{\mathrm{a}}$ \\
\hline & \multirow{8}{*}{ Current classification } & Domain Bacteria & TAS [9] \\
\hline & & Phylum Firmicutes & TAS [10-12] \\
\hline & & Class Clostridia & TAS $[13,14]$ \\
\hline & & Order Clostridiales & TAS $[15,16]$ \\
\hline & & Family XI Incertae sedis & TAS $[15,16]$ \\
\hline & & Genus Peptoniphilus & TAS [5] \\
\hline & & Species Peptoniphilus timonensis & IDA \\
\hline & & Type strain JC401 ${ }^{\top}$ & IDA \\
\hline & Gram stain & Positive & IDA \\
\hline & Cell shape & Coccoid & IDA \\
\hline & Motility & Nonmotile & IDA \\
\hline & Sporulation & Nonsporulating & IDA \\
\hline & Temperature range & Mesophile & IDA \\
\hline & Optimum temperature & $37^{\circ} \mathrm{C}$ & IDA \\
\hline MIGS-6.3 & Salinity & Growth in $\mathrm{BHI}$ medium $+1 \% \mathrm{NaCl}$ & IDA \\
\hline \multirow[t]{3}{*}{ MIGS-22 } & Oxygen requirement & Anaerobic & IDA \\
\hline & Carbon source & Unknown & NAS \\
\hline & Energy source & Unknown & NAS \\
\hline MIGS-6 & Habitat & Human gut & IDA \\
\hline \multirow[t]{3}{*}{ MIGS-15 } & Biotic relationship & Free living & IDA \\
\hline & Pathogenicity & Unknown & \\
\hline & Biosafety level & 2 & \\
\hline MIGS-14 & Isolation & Human feces & NAS \\
\hline MIGS-4 & Geographic location & Senegal & IDA \\
\hline MIGS-5 & Sample collection time & September 2010 & IDA \\
\hline MIGS-4.1 & Latitude & 13.7167 & IDA \\
\hline MIGS-4.1 & Longitude & -16.4167 & IDA \\
\hline MIGS-4.3 & Depth & Surface & IDA \\
\hline MIGS-4.4 & Altitude & $51 \mathrm{~m}$ above sea level & IDA \\
\hline
\end{tabular}

Evidence codes - IDA: Inferred from Direct Assay; TAS: Traceable Author Statement (i.e., a direct report exists in the literature); NAS: Non-traceable Author Statement (i.e., not directly observed for the living, isolated sample, but based on a generally accepted property for the species, or anecdotal evidence). These evidence codes are from the Gene Ontology project [17]. If the evidence is IDA, then the property was directly observed for a live isolate by one of the authors or an expert mentioned in the acknowledgements. 


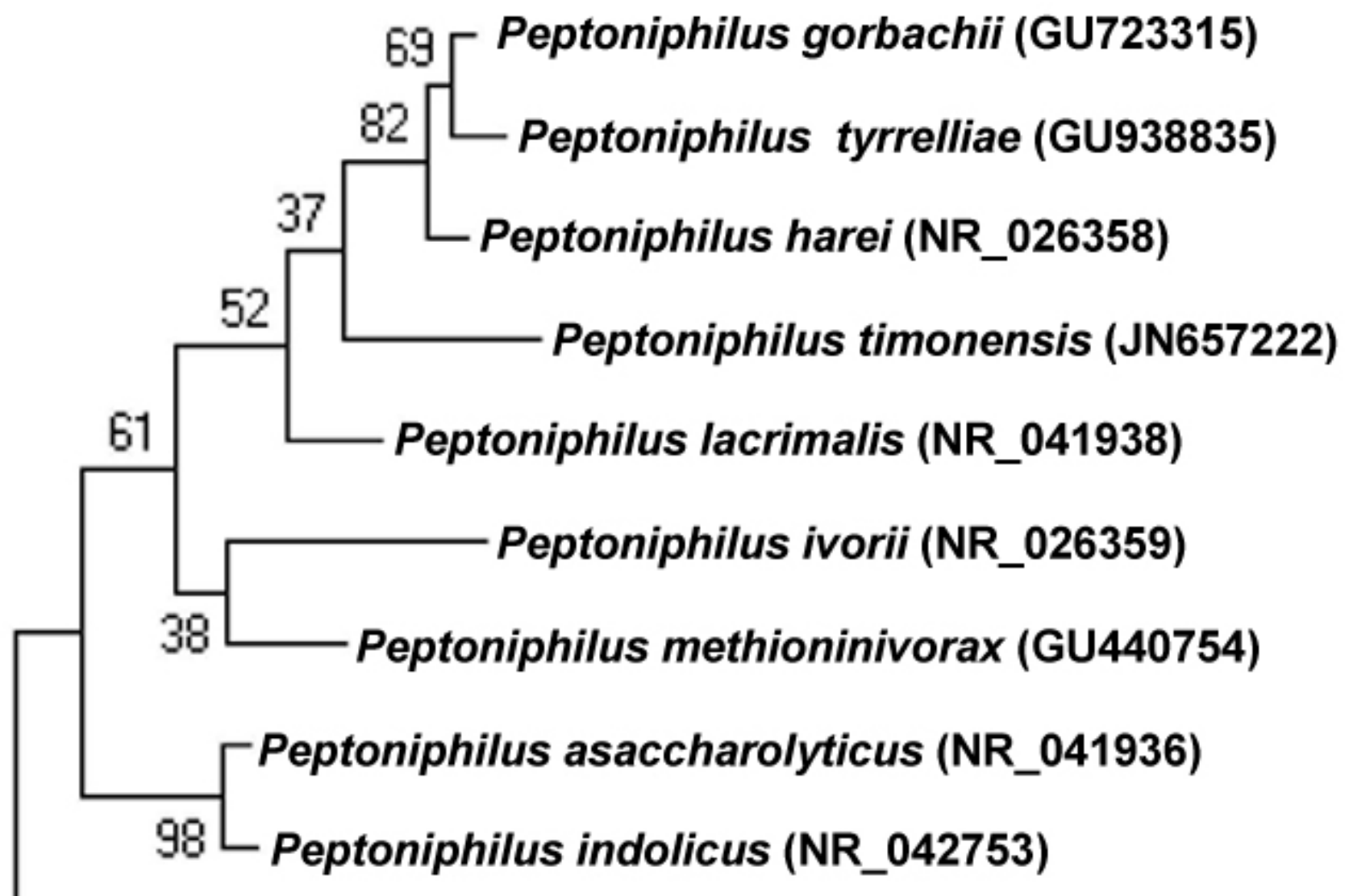

Anaerococcus lactolyticus (NR_041940)

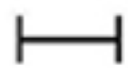

Figure 1. Phylogenetic tree highlighting the position of Peptoniphilus timonensis strain $\mathrm{JC} 401^{\top}$ relative to other type strains within the Peptoniphilus genus. GenBank accession numbers are indicated in parentheses. Sequences were aligned using CLUSTALW, and phylogenetic inferences obtained using the maximum-likelihood method within the MEGA software. Numbers at the nodes are percentages of bootstrap values obtained by repeating the analysis 500 times to generate a majority consensus tree. Anaerococcus prevotii was used as outgroup. The scale bar represents a $2 \%$ nucleotide sequence divergence.

Different growth temperatures $\left(25,30,37,45^{\circ} \mathrm{C}\right)$ were tested. Growth was not observed at $25^{\circ} \mathrm{C}$ and $45^{\circ} \mathrm{C}$, but optimal growth occurred between 30 and $37^{\circ} \mathrm{C}$. Colonies were $0.5 \mathrm{~mm}$ in diameter on blood-enriched BHI agar. Growth of the strain was tested under anaerobic and microaerophilic conditions using GENbag anaer and GENbag microaer systems, respectively (BioMérieux), and in aerobic conditions, with or without $5 \% \mathrm{CO}_{2}$. Growth was not achieved in aerobic (with and without $\mathrm{CO}_{2}$ ) conditions. The growth was observed in anaerobic conditions. Gram staining showed Gram-positive cocci (Figure 2). A motility test was negative. Cells grown on agar are sporulated and have a mean diameter of $0.91 \mu \mathrm{m}$ (Figure 3 ).

Strain JC401 ${ }^{\mathrm{T}}$ exhibited a catalase activity but no oxidase activity. Using API Rapid ID 32A, positive reactions were obtained for $\alpha$ galactosidase, arginine arylimidase, tyrosine arylamidase, histidine arylamidase, serine arylamidase and indole production. Weak reactions were observed for leucine arylamidase and phenylalanine arylamidase. $P$. timonensis is susceptible to penicillin $\mathrm{G}$, imipeneme, amoxicillin + clavulanic acid, vancomycin, clindamycin and metronidazole. 


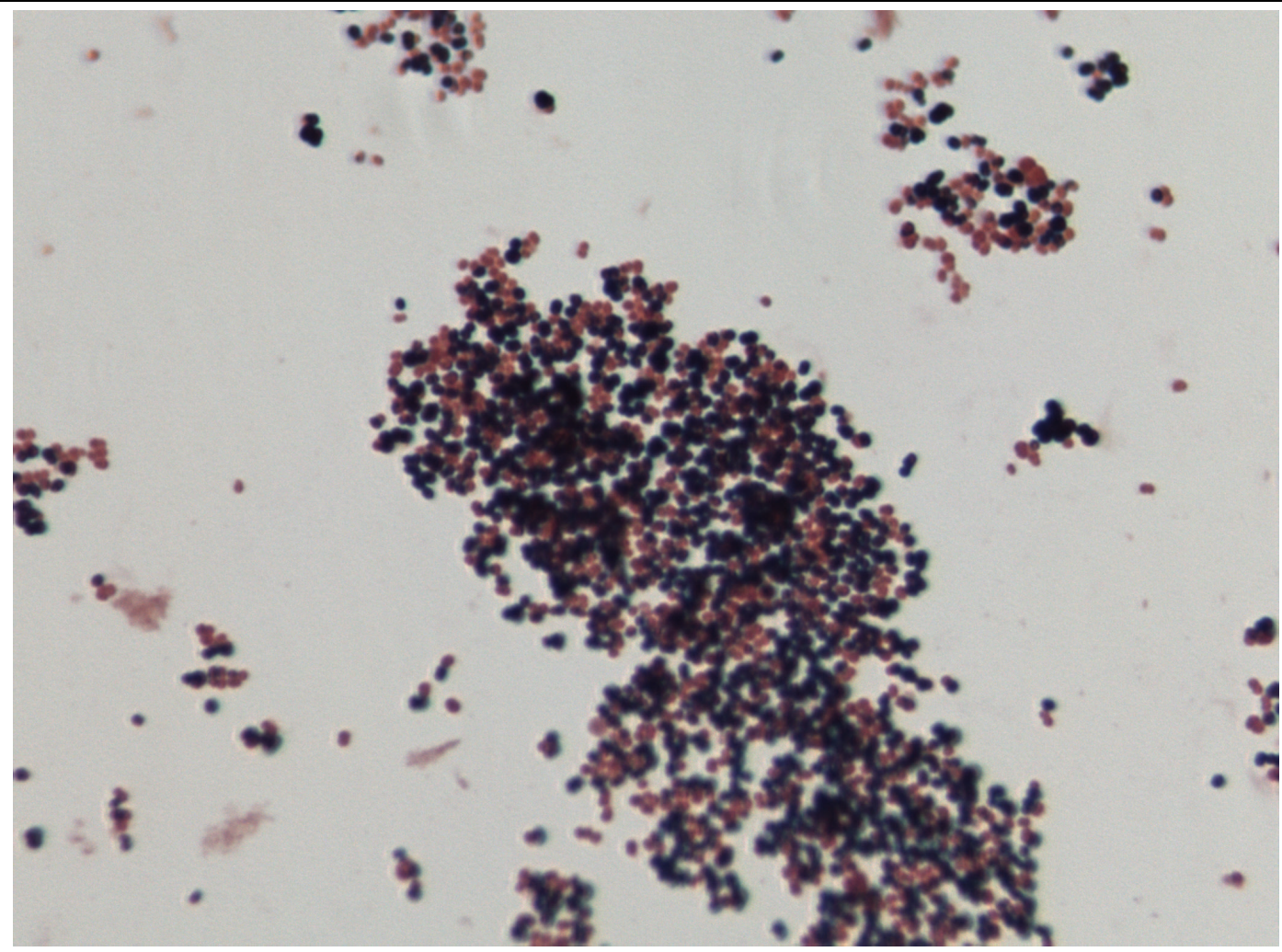

Figure 2. Gram staining of $P$. timonensis strain $\mathrm{JC} 401^{\mathrm{T}}$

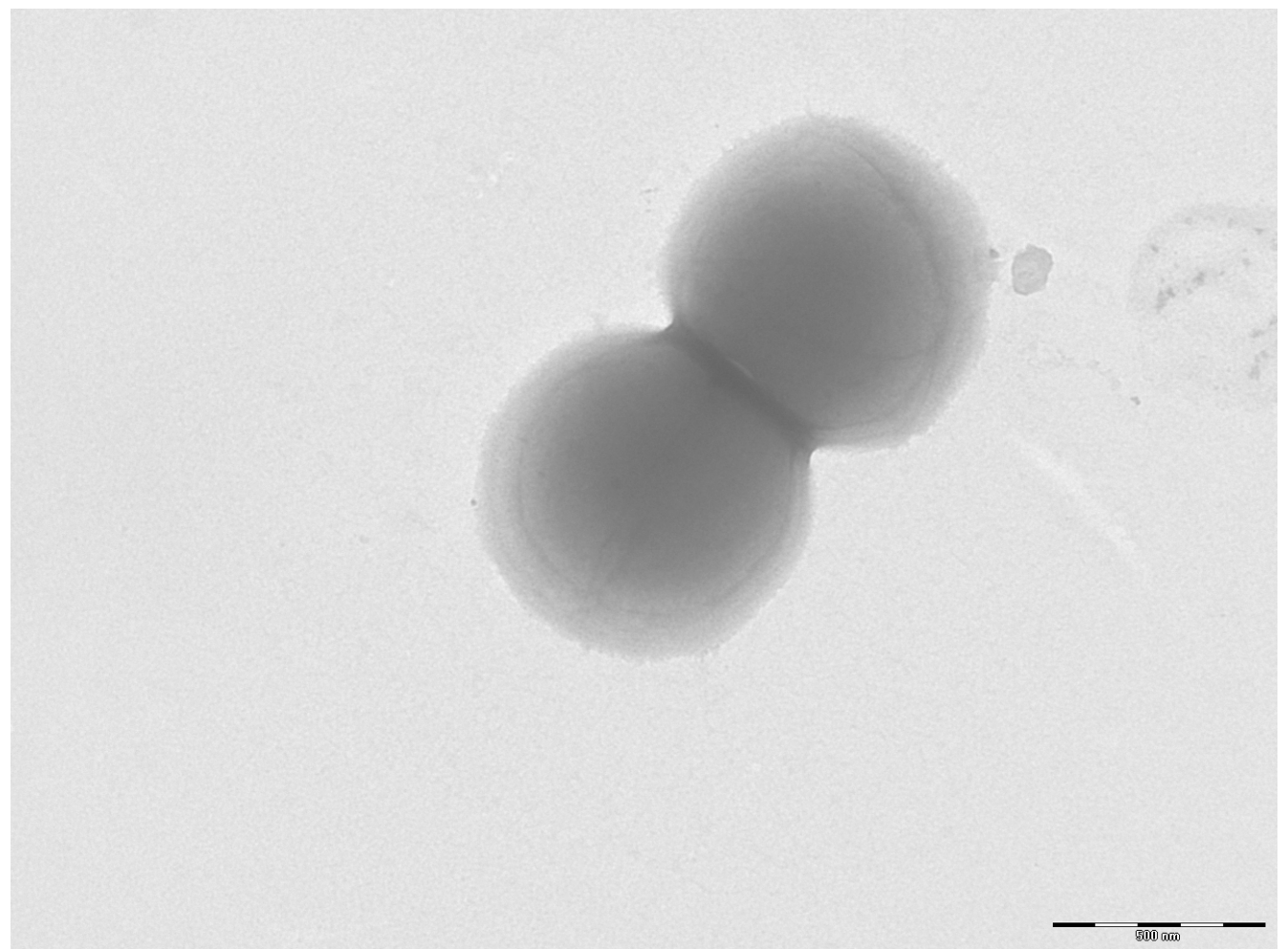

Figure 3. Transmission electron microscopy of $P$. timonensis strain $\mathrm{JC} 401^{\top}$, using a Morgani 268D (Philips) at an operating voltage of $60 \mathrm{kV}$. The scale bar represents $900 \mathrm{~nm}$. 
Matrix-assisted laser-desorption/ionization timeof-flight (MALDI-TOF) MS protein analysis was carried out as previously described [19]. Briefly, a pipette tip was used to pick one isolated bacterial colony from a culture agar plate, and to spread it as a thin film on an MTP 384 MALDI-TOF target plate (Bruker Daltonics, Leipzig, Germany). Twelve distinct deposits were done for strain JC401 ${ }^{\mathrm{T}}$ from twelve isolated colonies. Each smear was overlaid with $2 \mu \mathrm{L}$ of matrix solution (saturated solution of alpha-cyano-4-hydroxycinnamic acid) in 50\% acetonitrile, $2.5 \%$ tri-fluoraceticacid, and allowed to dry for five minutes. Measurements were performed with a Microflex spectrometer (Bruker). Spectra were recorded in the positive linear mode for the mass range of 2,000 to $20,000 \mathrm{Da}$ (parameter settings: ion source 1 (IS1), $20 \mathrm{kV}$; IS2, $18.5 \mathrm{kV}$; lens, $7 \mathrm{kV}$ ). A spectrum was obtained after 675 shots at a variable laser power. The time of acquisition was between 30 seconds and 1 minute per spot. The twelve JC401 spectra were imported into the MALDI BioTyper software (version 2.0, Bruker) and analyzed by standard pattern matching (with default parameter settings) against the main spectra of 3,769 bacteria, including 12 spectra from 8 Peptoniphilus species, which were used as reference data, in the BioTyper database. The method of identification included the $\mathrm{m} / \mathrm{z}$ from 3,000 to $15,000 \mathrm{Da}$. For every spectrum, 100 peaks at most were taken into account and compared with spectra in the database. A score enabled the identification, or not, from the tested species: a score $>2$ with a validated species enabled the identification at the species level, a score $>1.7$ but $<2$ enabled the identification at the genus level; and a score $<1.7$ did not enable any identification. For strain JC401' ${ }^{\mathrm{T}}$, the obtained score was 1.2, thus suggesting that our isolate was not a member of a known species. We incremented our database with the spectrum from strain JC401 ${ }^{\mathrm{T}}$ (Figure 4). The spectrum was made available online in our free-access URMS database [20].

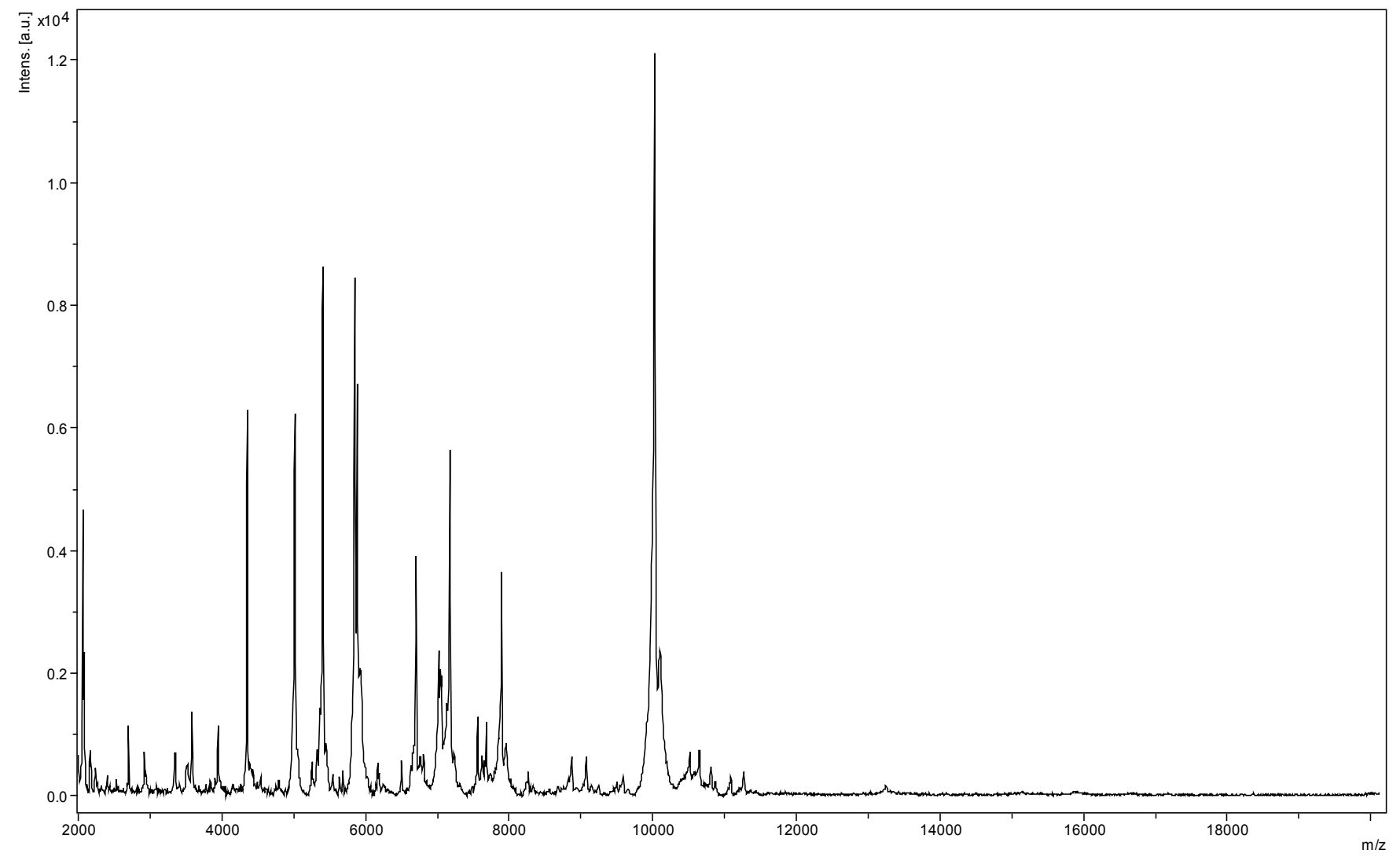

Figure 4: Reference mass spectrum from $P$. timonensis strain $\mathrm{JC} 401^{\top}$. Spectra from 12 individual colonies were compared and a reference spectrum was generated. 


\section{Genome sequencing information \\ Genome project history}

The organism was selected for sequencing on the basis of its phylogenetic position and 16S rRNA similarity to other members of the Peptoniphilus genus, and is part of a "culturomics" study of the human digestive flora aiming at isolating all bacterial species within human feces. It was the seventh genome of a Peptoniphilus species and the first genome of
Peptoniphilus timonensis sp. nov. The Genbank accession number is CAEL00000000 and consists of 97 large contigs. Table 2 shows the project information and its association with MIGS version 2.0 compliance.

Table 2. Project information

\begin{tabular}{lll}
\hline MIGS ID & Property & Term \\
\hline MIGS-31 & Finishing quality & High-quality draft \\
MIGS-28 & Libraries used & One 454 paired end 3-kb library \\
MIGS-29 & Sequencing platforms & 454 GS FLX Titanium \\
MIGS-31.2 & Fold coverage & 35 \\
MIGS-30 & Assemblers & Newbler version 2.5.3 \\
MIGS-32 & Gene calling method & Prodigal \\
& Gold ID & Gi16876 \\
& INSDC ID & PRJEB31 \\
& NCBI project ID & CAEL00000000 \\
& Genbank Date of Release & January 30, 2012 \\
& Project relevance & Study of the human gut microbiome \\
\hline
\end{tabular}

\section{Growth conditions and DNA isolation}

P. timonensis sp. nov. strain JC401 ${ }^{\mathrm{T}}$ (CSUR P165, DSM 25367) was grown anaerobically on 5\% sheep blood-enriched Columbia agar at $37^{\circ} \mathrm{C}$. Six petri dishes were spread and resuspended in $6 \times 100 \mu$ l of G2 buffer (EZ1 DNA Tissue kit, Qiagen). A first mechanical lysis was performed by glass powder on the Fastprep-24 device (Sample Preparation system, MP Biomedicals, USA) during 2x20 seconds. DNA was then treated with $2.5 \mu \mathrm{g} / \mu \mathrm{L}$ lysozyme $\left(30\right.$ minutes at $\left.37^{\circ} \mathrm{C}\right)$ and extracted using the BioRobot EZ1 Advanced XL (Qiagen). The DNA was then concentrated and purified using the Qiamp kit (Qiagen). The yield and the concentration was measured by the Quant-it Picogreen kit
(Invitrogen) on the Genios Tecan fluorometer at $123.3 \mathrm{ng} / \mu \mathrm{l}$.

\section{Genome sequencing and assembly}

Five $\mu \mathrm{g}$ of DNA was mechanically fragmented on the Hydroshear device (Digilab, Holliston, MA,USA) with an enrichment size at 3-4kb. The DNA fragmentation was visualized through the Agilent 2100 BioAnalyzer on a DNA labchip 7500 with an optimal size of $2.47 \mathrm{~kb}$. The library was constructed according to the 454 GS FLX Titanium paired end protocol. Circularization and nebulization were performed. After PCR amplification through 15 cycles followed by double size selection, the single stranded paired end library profile was visualized on an Agilent 2100 RNA Pico 6000 
Labchip with an optimal at 568bp. Then the library was quantified on the Quant-it Ribogreen kit (Invitrogen) on the Genios_Tecan fluorometer at 890 $\mathrm{pg} / \mu \mathrm{L}$. The library concentration equivalence was calculated as $2.87 \mathrm{E}+09$ molecules/ $\mu \mathrm{L}$. The library was stored at $-20^{\circ} \mathrm{C}$ until further use.

The shotgun library was clonal amplified with 0.25 and $0.5 \mathrm{cpb}$ in 2 emPCR reactions per conditions with the GS Titanium SV emPCR Kit (Lib-L) v2.The yields of the emPCR were $2.79 \%$ and $10.79 \%$ respectively in the range of 5 to $20 \%$ from the Roche procedure.

Approximately 790,000 beads for a $1 / 4$ region and 340000 beads for a $1 / 8$ region were loaded on the GS Titanium PicoTiterPlate PTP Kit $70 \times 75$ and sequenced with the GS FLX Titanium Sequencing Kit XLR70 (Roche). The run was performed overnight and then analyzed on the cluster through the gsRunBrowser and Newbler assembler (Roche). For the shotgun sequencing, 193,186 passed filter wells were obtained and generated $37.47 \mathrm{Mb}$ with a length average of $190 \mathrm{bp}$. The passed filter sequences were assembled Using Newbler with 90\% identity and $40 \mathrm{bp}$ as overlap. The final assembly identified 7 scaffolds and 97 large contigs (>1500bp) generating a genome size of $1.76 \mathrm{Mb}$.

\section{Genome annotation}

Open Reading Frames (ORFs) were predicted using Prodigal [21] with default parameters but the predicted ORFs were excluded if they were spanning a sequencing gap region. The predicted bacterial protein sequences were searched against the GenBank database [22] and the Clusters of Orthologous Groups (COG) databases using BLASTP. The tRNAScanSE tool [23] was used to find tRNA genes, whereas ribosomal RNAs were found by using RNAmmer [24] and BLASTn against the GenBank database. ORFans were identified if their BLASTP $E$-value was lower than 1e-03 for alignment length greater than 80 amino acids. If alignment lengths were smaller than 80 amino acids, we used an $E$ value of 1e-05. Such parameter thresholds have already been used in previous works to define ORFans.

To estimate the mean level of nucleotide sequence similarity at the genome level between Peptoniphilus species, we compared the ORFs only using BLASTN and the following parameters: query coverage of $\geq 70 \%$ and a minimum nucleotide length of $100 \mathrm{bp}$.

\section{Genome properties}

The genome is 1,758,598 bp long ( 1 chromosome, but no plasmid) with a $30.70 \%$ GC content (Figure 5 and Table 3). Of the 1,944 predicted genes, 1,922 were protein-coding genes and 22 were RNAs. A total of 1,368 genes $(70.37 \%)$ were assigned a putative function. A total of 186 genes were identified as ORFans (9.6\%). The remaining genes were annotated as hypothetical proteins. The distribution of genes into COGs functional categories is presented in Table 4. The properties and the statistics of the genome are summarized in Tables 3 and 4.

\section{Comparison with the genomes from other Peptoniphilus species}

Draft genome sequences are currently available for six species. Here we compared the genome sequence of $P$. timonensis strain $\mathrm{JC} 401^{\mathrm{T}}$ with those of $P$. harei strain ACS-146-V-Sch2b, $P$. indolicus strain ATCC BAA-1640 and P. lacrimalis strain 315-B.

The draft genome sequence of $P$. timonensis is larger than $P$. lacrimalis $(1.76 \mathrm{Mb}$ and $1.69 \mathrm{Mb}$, respectively) and smaller than $P$. indolicus and $P$. harei (2.2 $\mathrm{Mb}$ and $1.8 \mathrm{Mb}$, respectively). The $\mathrm{G}+\mathrm{C}$ content of $P$. timonensis is comparable to $P$. lacrimalis (30.7 and $29.91 \%$ respectively) but smaller than those of $P$. indolicus and $P$. harei (32.29 and $34.44 \%$ respectively). Additionally, $P$. timonensis has more predicted genes than $P$. harei and $P$. lacrimalis $(1,922,1,724$ and 1,589 respectively) and lesser than $P$. indolicus $(2,269)$. The genes assigned to COGs of $P$. timonensis are comparable to $P$. harei $(1,368$ and 1,381 respectively) greater than $P$. lacrimalis $(1,192)$ and lesser than $P$. indolicus $(1,690)$. However, the distribution of genes into COG categories (Table 4) was almost similar in all the four genomes.

In addition, $P$. timonensis shared a mean $86.49 \%$ (range 77.75 to $99.15 \%$ ), $85.54 \%$ (range 77.36 to 99.13) and $82.80 \%$ (range 77.43 to 95.39) sequence similarity with $P$. harei, $P$. lacrimalis and $P$. indolicus respectively at the genome level. 


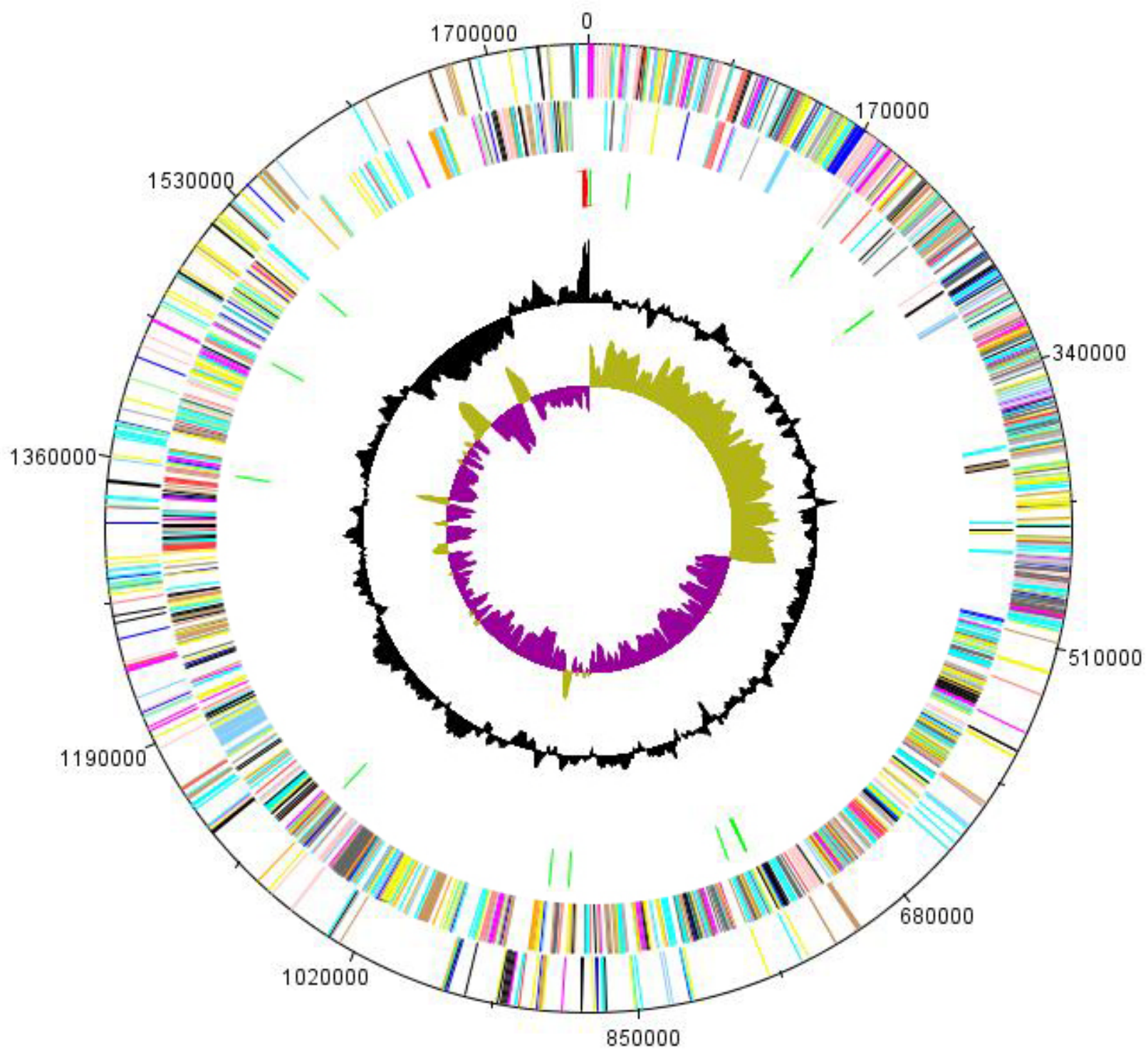

Figure 5. Graphical circular map of the chromosome. From outside to the center: Genes on the forward strand (colored by COG categories), genes on the reverse strand (colored by COG categories), RNA genes (tRNAs green, rRNAs red), GC content, and GC skew.

\section{Conclusion}

On the basis of phenotypic, phylogenetic and genomic analyses, we formally propose the creation of Peptoniphilus timonensis sp. nov. that contains the strain JC401' This strain has been found in Senegal.

\section{Description of Peptoniphilus timonensis sp. nov.}

Peptoniphilus timonensis (tim.on.en'sis. L. gen. masc. n. timonensis, of Timone, the name of the hospital where strain JC401T was cultivated. Isolated from stool from an asymptomatic Senegalese patient. P. timonensis is an anaerobic Gram-positive bacterium. Grows on axenic medium at $37^{\circ} \mathrm{C}$ in an anaerobic atmosphere. Strain JC401 ${ }^{\mathrm{T}}$ exhibited a catalase activity but no oxidase activity. Positive reactions were obtained for $\alpha$ galactosidase, arginine arylimidase, tyrosine arylamidase, histidine arylamidase, serine arylamidase and indole production. Weak reactions were observed for leucine arylamidase and phenylalanine arylamidase. Positive for indole. P. timonensis is susceptible to penicillin G, imipeneme, amoxicillin + clavulanic acid, vancomycin, clindamycin and metronidazole. Nonmotile. The $\mathrm{G}+\mathrm{C}$ content of the genome is $30.7 \%$. The type strain is $\mathrm{JC} 401^{\mathrm{T}}(=$ CSUR P165 $=$ DSM 25367). 
Table 3. Nucleotide content and gene count levels of the genome

\begin{tabular}{lrr}
\hline Attribute & Value & \% of total \\
\hline Genome size (bp) & $1,758,598$ & \\
DNA coding region (bp) & $1,566,468$ & 89.07 \\
DNA G+C content (bp) & $5,398,89$ & 30.7 \\
Total genes & 1,944 & 100 \\
RNA genes & 22 & 1.13 \\
Protein-coding genes & 1,922 & 98.87 \\
Genes with function prediction & 1,343 & 69.08 \\
Genes assigned to COGs & 1,368 & 70.37 \\
Genes with peptide signals & 119 & 6.12 \\
Genes with transmembrane helices & 450 & 23.15 \\
\hline
\end{tabular}

${ }^{a}$ The total is based on either the size of the genome in base pairs or the total number of protein coding genes in the annotated genome

Table 4. Number of genes associated with the 25 general COG functional categories

\begin{tabular}{crrl}
\hline Code & Value & \%age $^{\mathbf{a}}$ & Description \\
\hline J & 140 & 7.28 & Translation \\
A & 0 & 0 & RNA processing and modification \\
K & 101 & 5.25 & Transcription \\
L & 128 & 6.66 & Replication, recombination and repair \\
B & 1 & 0.05 & Chromatin structure and dynamics \\
D & 24 & 1.25 & Cell cycle control, mitosis and meiosis \\
Y & 0 & 0 & Nuclear structure \\
V & 47 & 2.45 & Defense mechanisms \\
T & 62 & 3.23 & Signal transduction mechanisms \\
M & 66 & 3.43 & Cell wall/membrane biogenesis \\
N & 5 & 0.26 & Cell motility \\
Z & 0 & 0 & Cytoskeleton \\
W & 0 & 0 & Extracellular structures \\
U & 26 & 1.35 & Intracellular trafficking and secretion \\
O & 56 & 2.91 & Posttranslational modification, protein turnover, chaperones \\
C & 79 & 4.11 & Energy production and conversion \\
G & 40 & 2.08 & Carbohydrate transport and metabolism \\
E & 137 & 7.13 & Amino acid transport and metabolism \\
F & 61 & 3.17 & Nucleotide transport and metabolism \\
H & 58 & 3.02 & Coenzyme transport and metabolism \\
I & 41 & 2.13 & Lipid transport and metabolism \\
P & 77 & 4.01 & Inorganic ion transport and metabolism \\
Q & 15 & 0.78 & Secondary metabolites biosynthesis, transport and catabolism \\
R & 179 & 9.31 & General function prediction only \\
S & 133 & 6.92 & Function unknown \\
- & 554 & 28.82 & Not in COGs \\
\hline & & &
\end{tabular}

${ }^{a}$ The total is based on the total number of protein coding genes in the annotated genome 


\section{References}

1. Rossello-Mora R. DNA-DNA Reassociation Methods Applied to Microbial Taxonomy and Their Critical Evaluation. In: Stackebrandt E (ed), Molecular Identification, Systematics, and population Structure of Prokaryotes. Springer, Berlin, 2006, p. 23-50.

2. Stackebrandt E, Ebers J. Taxonomic parameters revisited: tarnished gold standards. Microbiol Today 2006; 33:152-155.

3. Welker M, Moore ER. Applications of whole-cell matrix-assisted laser-desorption/ionization timeof-flight mass spectrometry in systematic microbiology. Syst Appl Microbiol 2011; 34:2-11. PubMed

$\underline{\text { http://dx.doi.org/10.1016/j.syapm.2010.11.013 }}$

4. Tindall BJ, Rosselló-Móra R, Busse HJ, Ludwig W, Kämpfer P. Notes on the characterization of prokaryote strains for taxonomic purposes. Int I Syst Evol Microbiol 2010; 60:249-266. PubMed http://dx.doi.org/10.1099/ijs.0.016949-0

5. Ezaki T, Kawamura Y, Li N, Li ZY, Zhao L, Shu S. Proposal of the genera Anaerococcus gen. nov., Peptoniphilus gen. nov. and Gallicola gen. nov. for members of the genus Peptostreptococcus. Int J Syst Evol Microbiol 2001; 51:1521-1528. PubMed

6. Song Y, Liu C, Finegold SM. Peptoniphilus gorbachii sp. nov., Peptoniphilus olsenii sp. nov. and Anaerococcus murdochii sp. nov. isolated from clinical specimens of human origin. J Clin Microbiol 2007; 45:1746-1752. PubMed http://dx.doi.org/10.1128/JCM.00213-07

7. Rooney AP, Swezey JL, Pukall R, Schumann P, Spring S. Peptoniphilus methioninivorax sp. nov., a Gram-positive anaerobic coccus isolated from retail ground beef. Int / Syst Evol Microbiol 2011; 61:1962-1967. PubMed http://dx.doi.org/10.1099/ijs.0.024232-0

8. Field D, Garrity G, Gray T, Morrison N, Selengut J, Sterk P, Tatusova T, Thomson N, Allen MJ, Angiuoli SV, et al. The minimum information about a genome sequence (MIGS) specification. Nat Biotechnol 2008; 26:541-547. PubMed http://dx.doi.org/10.1038/nbt1360

9. Woese CR, Kandler O, Wheelis ML. Towards a natural system of organisms: proposal for the domains Archae, Bacteria, and Eukarya. Proc Natl Acad Sci USA 1990; 87:4576-4579. PubMed http://dx.doi.org/10.1073/pnas.87.12.4576
10. Gibbons NE, Murray RGE. Proposals Concerning the Higher Taxa of Bacteria. Int I Syst Bacteriol 1978; 28:1-6.

http://dx.doi.org/10.1099/00207713-28-1-1

11. Garrity GM, Holt JG. The Road Map to the Manual. In: Garrity GM, Boone DR, Castenholz RW (eds), Bergey's Manual of Systematic Bacteriology, Second Edition, Volume 1, Springer, New York, 2001, p. 119-169.

12. Murray RGE. The Higher Taxa, or, a Place for Everything...? In: Holt JG (ed), Bergey's Manual of Systematic Bacteriology, First Edition, Volume 1, The Williams and Wilkins Co., Baltimore, 1984, p. 31-34.

13. List Editor. List of new names and new combinations previously effectively, but not validly, published. List no. 132. Int J Syst Evol Microbiol 2010; 60:469-472. http://dx.doi.org/10.1099/ijs.0.022855-0

14. Rainey FA. Class II. Clostridia class nov. In: De Vos P, Garrity G, Jones D, Krieg NR, Ludwig W, Rainey FA, Schleifer KH, Whitman WB (eds), Bergey's Manual of Systematic Bacteriology, Second Edition, Volume 3, Springer-Verlag, New York, 2009, p. 736.

15. Skerman VBD, Sneath PHA. Approved list of bacterial names. Int J Syst Bact 1980; 30:225-420. http://dx.doi.org/10.1099/00207713-30-1-225

16. Prevot AR. Dictionnaire des bactéries pathogens. In: Hauduroy P, Ehringer G, Guillot G, Magrou J, Prevot AR, Rosset, Urbain A (eds). Paris, Masson, 1953, p.1-692.

17. Ashburner M, Ball CA, Blake JA, Botstein D, Butler H, Cherry JM, Davis AP, Dolinski K, Dwight SS, Eppig JT, et al. Gene ontology: tool for the unification of biology. The Gene Ontology Consortium. Nat Genet 2000; 25:25-29. PubMed http://dx.doi.org/10.1038/75556

18. Stackebrandt E, Ebers J. Taxonomic parameters revisited: tarnished gold standards. Microbiol Today 2006; 33:152-155.

19. Seng P, Drancourt M, Gouriet F, La Scola B, Fournier PE, Rolain JM, Raoult D. Ongoing revolution in bacteriology: routine identification of bacteria by matrix-assisted laser desorption ionization time-of-flight mass spectrometry. Clin Infect Dis 2009; 49:543-551. PubMed http://dx.doi.org/10.1086/600885 
Mishra et al.

20. URMS database. http://ifr48.timone.univmrs.fr/portail2/index.php ? op$\underline{\text { tion}=\text { com } c o n t e n t \& \text { task=view }}$

21. Prodigal. http://prodigal.ornl.gov/

22. Benson DA, Karsch-Mizrachi I, Clark K, Lipman DJ, Ostell J, Sayers EW. GenBank. Nucleic Acids Res 2012; 40:D48-D53. PubMed

http://dx.doi.org/10.1093/nar/gkr1202
23. Lowe TM, Eddy SR. t-RNAscan-SE: a program for imroved detection of transfer RNA gene in genomic sequence. Nucleic Acids Res 1997; 25:955-964. PubMed

24. Lagesen K, Hallin P, Rodland EA, Staerfeldt HH, Rognes T, Ussery DW. RNAmmer: consistent and rapid annotation of ribosomal RNA genes. Nucleic Acids Res 2007; 35:3100-3108. PubMed http://dx.doi.org/10.1093/nar/gkm160 